\title{
ESTRESSE E PROFISSIONAIS DA SAÚDE: PRODUÇÃO DO CONHECIMENTO NO CENTRO DE ENSINO E PESQUISAS EM ENFERMAGEM
}

\author{
Graciele Fernanda da Costa Linch ${ }^{1}$, Laura de Azevedo Guido², Juliane Umann ${ }^{3}$
}

\begin{abstract}
RESUMO: Trata-se de uma revisão de literatura com objetivo de identificar publicações que adotam o estresse como tema de pesquisa e realizam relação deste com a saúde do trabalhador. Foi realizada nos catálogos do Centro de Ensino e Pesquisas em Enfermagem (Epen), no período de 1979 a 2007, sendo incluídas teses e dissertações e excluídos os trabalhos de revisão de literatura ou interativa ou estudos que tiveram como sujeitos pacientes e familiares. Desta maneira, foram selecionados 30 trabalhos. Verificou-se que nos últimos dez anos houve maior número de produções relacionadas ao tema. Conclui-se que os estudos são recentes, em sua maioria, descritivos, com a identificação de estressores e levantamento da relação causal. Salienta-se a necessidade da continuidade de estudos que envolvam situações e sentimentos dos profissionais de saúde relacionados ao trabalho e saúde.
\end{abstract}

PALAVRAS-CHAVE: Enfermagem; Estresse; Trabalho.

\section{STRESS AND HEALTH PROFESSIONALS: PRODUCTION OF KNOWLEDGE IN THE CENTER OF EDUCATION AND RESEARCH IN NURSING}

\begin{abstract}
This is a literature review in order to identify publications that take stress as a research topic and conduct its relation to occupational health. It was performed in the catalogs of the Center for Teaching and Research in Nursing (Epen) in the period from 1979 to 2007, and included theses and dissertations and excluded the review work of literature or interactive or studies that had patients and their families as subjects. Thus, we selected 30 works. It was found that in the last ten years there have been many productions with this subject. It is concluded that the studies are recent, mostly descriptive, with the identification of stressors and withdrawal of the causal relationship. The need for further studies involving situations and feelings of health professionals and health-related work is stressed.
\end{abstract}

KEYWORDS: Nursing; Stress; Work.

\section{ESTRESE Y PROFESIONALES DE LA SALUD: PRODUCCIÓN DEL CONOCIMIENTO EN CENTRO DE ENSEÑANZA Y INVESTIGACIONES EN ENFERMERÍA}

RESUMEN: Es una revisión de literatura con objetivo de identificar publicaciones que adoptan el estrese como tema de investigación y realizan conexión entre este y la salud del trabajador. Fue realizada en los catálogos del Centro de Enseñanza y Investigaciones en Enfermería (Epen), en periodo de 1979 a 2007, siendo incluidas tesis y disertaciones y excluidos los trabajos de revisión de literatura o interativa o estudios que tuvieron como sujetos pacientes y familiares. De esta forma, fueron seleccionados 30 trabajos. Se verificó que, en los últimos diez años, hubo mayor número de producciones relacionadas al tema. Se concluye que los estudios son recientes, en su mayoría descriptivos, con la identificación de estresores y levantamiento de la relación causal. Se destaca la necesidad de la continuidad de estudios que abarquen situaciones y sentimientos de los profesionales de salud relacionados al trabajo y a la salud.

PALABRAS CLAVE: Enfermería; Estrese; Trabajo.

\footnotetext{
${ }^{1}$ Enfermeira. Mestre em Enfermagem. Doutoranda em Enfermagem pelo Programa de Pós-Graduação em Enfermagem na Universidade Federal do Rio Grande do Sul-UFRGS.

${ }^{2}$ Enfermeira. Doutora em Enfermagem. Professor Adjunto do Departamento de Enfermagem da Universidade Federal de Santa Maria-UFSM. Coordenadora Substituta do Programa de Pós-Graduação em Enfermagem-UFSM.

${ }^{3}$ Enfermeira. Mestranda do Programa de Pós-Graduação em Enfermagem da UFSM. Bolsista Capes. Membro do Grupo de Pesquisa Trabalho, Saúde, Educação e Enfermagem-UFSM.
}

Autor correspondente:

Graciele Fernanda da Costa Linch

Universidade Federal do Rio Grande do Sul

Rua Silva Jardim, 1899 - 97010-493 - Santa Maria-RS, Brasil

Recebido: 24/08/09

E-mail: gracielelinch@gmail.com

Aprovado: 26/06/10 


\section{INTRODUÇÃO}

No Brasil, as pesquisas desenvolvidas junto a programas de pós-graduação em Enfermagem relacionadas ao estresse e à saúde dos profissionais de saúde iniciaram-se na década de $90^{(1)}$. No entanto, foi por volta de 1936 que o termo estresse foi introduzido nas ciências biológicas por Hans Seyle, sendo ele o precursor deste tema na abordagem da teoria biologicista. Em continuidade às discussões de Seyle, desenvolveram-se diferentes modelos, como o modelo interacionista de Lazarus e Folkman ${ }^{(1-2)}$.

Pautados nestas teorias - interacionista e biologicista - e com o princípio de que o estresse pode apresentar-se como um risco ocupacional para os trabalhadores na área da saúde, pesquisadores objetivam identificar, avaliar, ou ainda, medir o nível de estresse dos profissionais de saúde, principalmente do enfermeiro e de sua equipe, como também investigar os estressores presentes no trabalho, em diversas unidades ou cenários, e sua relação com a saúde desses profissionais.

O Centro de Ensino e Pesquisas em Enfermagem (CEPEN) visa o desenvolvimento e a divulgação da pesquisa em Enfermagem, e a organização e preservação de documentos históricos dessa profissão. Disponibiliza catálogos com os resumos de pesquisas desenvolvidas desde 1979. Assim, constitui-se como o maior banco de teses e dissertações desta área no Brasil.

Desta maneira, delineou-se uma revisão de literatura com o objetivo de identificar, a partir dos catálogos do CEPEN, teses e dissertações publicadas que adotam o estresse como tema de pesquisa e analizam a relação deste com a saúde do trabalhador.

\section{MÉTODOS}

Trata-se de uma revisão de literatura realizada por meio de uma busca nos catálogos do CEPEN, no período de 1979 a 2007. Foram selecionados os estudos conforme os seguintes critérios: incluídas teses e dissertações que adotam o estresse como tema de pesquisa e abordam a relação deste com a saúde dos profissionais; e excluídos os trabalhos de revisão de literatura ou revisão interativa, bem como os estudos que tiveram como sujeitos pacientes e familiares.

Para o desenvolvimento da pesquisa foram percorridas as seguintes etapas: estabelecimento do objetivo; definição de critérios de inclusão e exclusão das teses e dissertações (seleção da amostra); apresentação e discussão dos resultados. A coleta de dados deu-se pelo índice de cada catálogo, sendo que os descritores utilizados foram: estresse e estresse psicológico. Para a organização dos dados foi elaborada uma planilha no Excel composta pelos seguintes itens: autores, título, ano, abordagem metodológica (quantitativa ou qualitativa), universidade, orientadores, categoria (tese ou dissertação) e sujeitos. Posteriormente, foi realizada a categorização considerando o enfoque principal do trabalho.

\section{RESULTADOS}

Foram encontrados 72 estudos (15 teses e 57 dissertações). Destes, e de acordo com os critérios previamente definidos, foram selecionadas 25 dissertações $(83,33 \%)$ e cinco teses $(16,67 \%)$, totalizando 30 trabalhos.

Em 1990, foi apresentado o primeiro traba1ho, a tese de doutorado de Bianchi ${ }^{(1)}$, pela Escola de Enfermagem da Universidade Estadual de São Paulo, intitulada: "Estresse em enfermagem: uma análise da atuação do enfermeiro de centro cirúrgico"(1). No entanto, verificou-se uma lacuna na produção de conhecimento no período de sete anos; sendo que nos últimos dez anos houve maior número de produções relacionadas ao tema, e nesse período, os anos de 2002 e 2006 reuniram a maior quantidade de trabalhos produzidos, sendo contabilizados seis para cada ano.

Em relação à abordagem metodológica utilizada, se qualitativa ou quantitativa, pode-se verificar, no gráfico 1 , que houve predomínio de pesquisas quantitativas, com um total de $23(76,66 \%)$ produções.

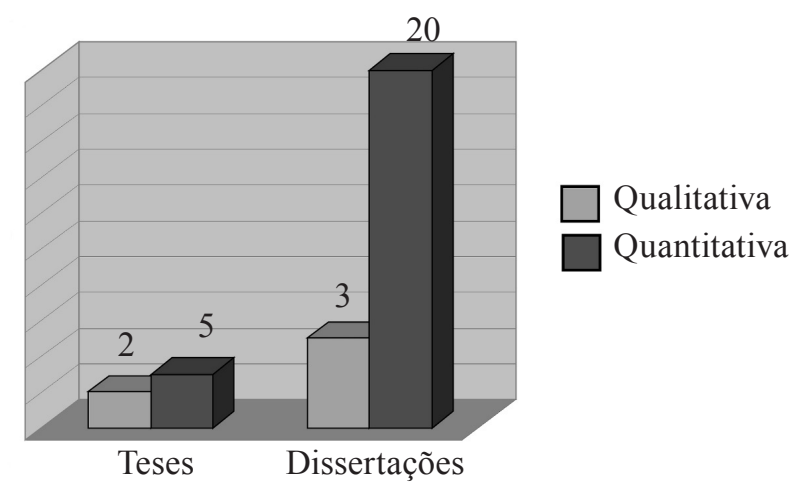

Fonte: Centro de Ensino e Pesquisas em Enfermagem.

Gráfico 1 - Abordagens metodológicas utilizadas em relação à categoria em teses e dissertações de Enfermagem. 1979-2007.

Apresentaram-se duas teses $(6,67 \%)$ e cinco dissertações $(16,67 \%)$ com abordagem qualitativa, e três teses $(10,00 \%)$ e 20 dissertações $(66,66 \%)$ com abordagem quantitativa.

Pode-se identificar que os estudos foram 
realizados junto a 12 programas de pós-graduação, são evidenciados, no gráfico 2, o programa de pósgraduação da Escola de Enfermagem da Universidade de São Paulo (EEUSP) com o maior número de estudos, contendo 10 produções (33,33\%), seguido pelo da Escola de Enfermagem de Ribeirão Preto (EEUSP/RP) com sete pesquisas (23,34\%), e ainda a Universidade Federal de Santa Catarina (UFSC) com três estudos (10,00\%), sendo os demais estudos distribuídos em outros 10 programas de diferentes instituições de ensino (33,33\%).

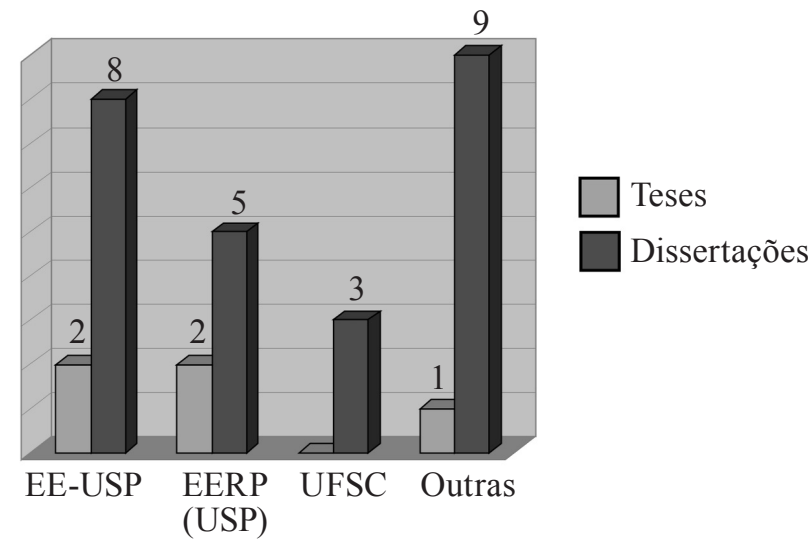

Fonte: Centro de Ensino e Pesquisas em Enfermagem.

Gráfico 2 - Teses e dissertações distribuídas nos Programas de Pós-Graduação em Enfermagem. 1979-2007.

Dentre os profissionais da saúde pesquisados, os enfermeiros apresentaram-se como sujeitos em 16 estudos (53,33\%), seguidos pela equipe de enfermagem $(33,34 \%)$, sendo o restante $(13,33 \%)$ desenvolvido com agentes comunitários de saúde e outros profissionais.

Quanto à unidade de trabalho dos pesquisados, verificou-se que em $25,80 \%$ dos estudos foram investigados sujeitos alocados em diferentes setores hospitalares. Destaca-se, como foco de algumas pesquisas, o centro cirúrgico com sete $(22,58 \%)$ e a emergência com cinco estudos (16,13\%), sendo o restante desenvolvido em outros setores hopitalares $(19,36 \%)$. Cabe salientar que cinco $(16,13 \%)$ pesquisas foram desenvolvidas em unidades básicas de saúde.

Verificou-se que a Escala Bianchi de Estresse foi utilizada em 8 pesquisas (26,67\%), o Inventário de Sintomas de Estresse de LIPP em quatro (13,33\%) e o Inventário de Ansiedade Traço-Estado em três (10,00\%). Os demais estudos (50\%) utilizaram outros instrumentos, ou ainda outros métodos para a investigação.

\section{DISCUSSÃO}

Pode-se considerar como precursora de estu- dos de estresse e enfermagem no Brasil a tese apresentada por Bianchi ${ }^{(1)}$. Nesse estudo, foi desenvolvida a Escala Bianchi de Estresse, instrumento que se propõe a identificar o nível de estresse dos profissionais e permite verificar situações consideradas estressantes no trabalho, e que passou a ser utilizado em estudos posteriores $^{(2-8)}$.

O ambiente hospitalar pode constituir um importante estressor para os profissionais devido aos possíveis sofrimentos vivenciados nesse local, às condições de trabalho, às demandas requeridas pela assistência, e também pela grande responsabilidade exigida no trabalho ${ }^{(2)}$. Salienta-se que, ao mesmo tempo, esse ambiente subdivide-se em unidades, as quais se caracterizam distintamente, o que pode influenciar diretamente o trabalho e a percepção deste para o trabalhador frente aos estressores.

O centro cirúrgico $(\mathrm{CC})$ foi o cenário de alguns estudos ${ }^{(1-2,9-13)}$ analisados nesta pesquisa, pois possui características peculiares, tais como: ser uma unidade fechada, de alta complexidade, com demanda de cuidados críticos, e ainda envolver situações de sofrimento, emergências, dentre outras. Tudo isso torna o ambiente propenso a insatisfação e desgaste e, portanto, justifica-se como cenário destas pesquisas.

$\mathrm{O}$ enfermeiro do $\mathrm{CC}$ se depara com muitos estressores no trabalho, destacando-se as situações envolvidas na coordenação de atividades, as condições de trabalho e a assistência a pacientes graves ${ }^{(1-2,9,12-13)}$. No entanto, esses trabalhadores utilizam estratégias de enfrentamento que viabilizam a realização de sua atividade profissional ${ }^{(2)}$.

Um estudo destaca a prevalência de estratégias de enfrentamento focadas no problema, pelos enfermeiros, sendo por eles considerada mais segura na medida em que oportuniza à pessoa ter experiência e amadurecimento com os recursos adaptativos ${ }^{(14)}$. E ainda, quando se investigou os sentimentos de prazer e sofrimento destes profissionais, encontrou-se uma díade, não antagônica, mas que compõe o processo de viver e trabalhar neste ambiente ${ }^{(10)}$. Os principais mecanismos utilizados para o enfrentamento do estresse estão focados na emoção e no problema.

Pesquisa que adotou o referencial teórico metodológico das Representações Sociais e teve como objetivo analisar a tríade estressor/estresse/enfrentamento pela equipe de enfermagem do $\mathrm{CC}$ apontou que esses profissionais reconhecem estar sob a influência do estresse no trabalho, e ressaltam como estressor as relações interpessoais, sendo o $\mathrm{CC}$ reconhecido como sua casa ${ }^{(11)}$. 
Estudo destaca que os trabalhadores de enfermagem identificam os estressores e os recursos compensatórios para reduzi-los, mas não associam seus problemas de saúde e doenças com as condições de trabalho, o que sugere um programa de educação para a saúde visando à conscientização desses profissionais $^{(15)}$. Outros estudos que investigaram a relação entre estresse e estado de saúde concluíram não haver correlações estatísticas significativas entre as duas variáveis ${ }^{(3-4)}$. Sobre esta questão, outra pesquisa revela que enfermeiros avaliam sua saúde como satisfatória, inferindo que o trabalho não é altamente estressante ${ }^{(16)}$.

No entanto, recentemente, outro estudo, partindo do estresse como fator relevante no processo de determinação de agravos à saúde, analisou a exposição ao estresse no trabalho, tendo como desfecho a ocorrência de transtornos mentais comuns (TMC) em trabalhadores de enfermagem ${ }^{(17)}$. Constatou-se a associação entre a exposição às dimensões de estresse e suspeição de TMC, sendo que os resultados apontam necessidades de intervenções na organização do trabalho, com o intuito de minimizar o estresse e promover a saúde mental. Ainda, existem evidências da necessidade de reorganização do trabalho como estratégia para minimizar a exposição das enfermeiras às cargas psíquicas geradoras de estresse ${ }^{(8)}$.

Nesse sentido, um pesquisador investigou a presença e o nível de estresse emocional, os sintomas físicos e psicológicos, e a intensidade do estresse em enfermeiros que fazem dupla jornada de trabalho e os que não o fazem ${ }^{(18)}$. Os sintomas mais citados foram: cansaço constante, sensação de desgaste físico constante, tensão muscular e irritabilidade sem causa aparente. $\mathrm{O}$ estudo ainda observou que os sujeitos estavam na fase de resistência, sendo que a maioria encontrava-se no nível médio de estresse. Quando comparados, os sujeitos com dupla jornada estavam mais estressados em relação aos sujeitos que realizavam jornada única.

Outro estudo buscou a correlação entre estresse, cronótipo e horário de trabalho ${ }^{(19)}$, tendo participado 87 enfermeiros hospitalares de diferentes setores e turnos de trabalho. A partir dos resultados, concluiuse que a maioria dos sujeitos estava adequada ao turno de trabalho, de acordo com seu cronótipo. Porém, o estresse foi observado em grande porcentagem da população, o que levou a confirmar o caráter estressante da profissão.

Por outro lado, estudo com abordagem qualitativa objetivou analisar as repercussões do estresse ocupacional na vida cotidiana do enfermeiro, em uma instituição hospitalar pública ${ }^{(20)}$. A autora identificou como principais estressores presentes no cotidiano de trabalho: as condições precárias de trabalho; a sobrecarga de trabalho e a excessiva jornada de trabalho. Quanto ao nexo causal entre o processo de trabalho e o adoecimento, encontraram-se, principalmente, queixas alusivas a problemas de coluna e a problemas cardiovasculares. Concluiu este estudo que o estresse ocupacional decorrente de um processo de trabalho hospitalar, marcado por condições precárias de trabalho e pelo aumento da jornada de trabalho, tem fortes repercussões no cotidiano profissional e pessoal das enfermeiras entrevistadas.

Nas unidades de Pronto Socorro (PS), realizam-se os atendimentos de urgências e emergências, sendo assim, os profissionais que atuam nestas unidades devem ser capazes de tomar decisões rápidas, precisas e capazes de distinguir as prioridades, devendo atender a demanda com muita agilidade, conhecimento e segurança. Essas situações de trabalho podem ser avaliadas como positivas, um desafio, ou como negativas, uma ameaça; em ambas, o estresse está presente ${ }^{(5-6)}$.

Em vista dos estressores identificados no trabalho da enfermagem em PS, alguns autores propõem alternativas gerenciais na estrutura organizacional e nas políticas para minimizar a intensidade do estresse, o que pode interferir na vida pessoal, profissional e no estado de saúde do indivíduo ${ }^{(5,21)}$. Outra proposta referiu-se à utilização de música erudita nesse ambiente, pois, quando avaliada essa intervenção, obteve-se benefícios aos profissionais ${ }^{(2)}$.

Em estudo realizado com auxiliares de enfermagem de um PS, objetivou identificar os estressores presentes no banho de aspersão $0^{(23)}$. A partir disto, foram sugeridos a implementação de ações como as mudanças na rotina hospitalar, visando a proteção dos funcionários e pacientes contra situações passíveis de modificação, e os cursos profissionalizantes com discussões a respeito dos procedimentos.

As unidades de terapia intensiva são destinadas à assistência a pacientes graves que necessitam de cuidados diretos e complexos, o que exige equipe qualificada e alta tecnologia, além de ser um ambiente fechado, com características próprias ${ }^{(7,24-25)}$. Essas peculiaridades podem favorecer o estresse dos profissionais que atuam nessas unidades. Estudos apontam ações preventivas, suporte social, alternativas de organização interna das unidades e medidas de gerenciamento de enfermagem como alternativas para minimizar os possíveis riscos relacionados ao estresse ${ }^{(24,26)}$. 
Alguns estudos foram realizados com trabalhadores de unidades básicas de saúde, impulsionados pelas recentes estratégias assumidas pelo Ministério da Saúde, que visam substituir o modelo tradicional da assistência à família ${ }^{(27-30)}$. Nestes, foram identificados como principais estressores: falta de autonomia, trabalho repetitivo, conflito no trabalho em equipe, falta de preparo e de capacitação ${ }^{(27,29)}$.

Pode-se perceber que o estresse tem sido investigado em diferentes cenários da atuação dos profissionais de saúde, em especial, no ambiente hospitalar. Porém, as pesquisas acerca do estresse entre os trabalhadores da atenção primária apresentam-se como um novo foco para os estudos. Da mesma forma, observa-se uma transição de estudos descritivos para analíticos.

\section{CONCLUSÕES}

Observou-se que a maior parte dos trabalhos teve fundamentação na corrente filosófica do positivismo. As teses e dissertações que abordam estresse e realizam a relação deste com a saúde do trabalhador de enfermagem são recentes, sendo que o número de estudos vem crescendo nos últimos anos. Este aumento pode estar associado à expansão, desenvolvimento e aumento de Programas de Pós-Graduação em Enfermagem.

Diante da análise destas pesquisas, pode-se verificar que, em sua maioria, elas encontram-se em uma fase inicial, são descritivas, identificando os estressores e levantando a relação causal. Essa fase é importante para identificar problemas que envolvem os profissionais de saúde no ambiente laboral, no intuito de solucioná-los, e ainda, serve de subsídio para uma atuação científica.

É fundamental para a Enfermagem o desenvolvimento e a continuidade de estudos que envolvam as situações e sentimentos dos profissionais de saúde relacionados ao trabalho e à sua saúde. É a partir destes estudos, embasados cientificamente, que se espera que os profissionais sejam estimulados e desenvolvam mecanismos para identificar, enfrentar e gerenciar os estressores no trabalho.

\section{REFERÊNCIAS}

1. Bianchi ERF. Estresse em enfermagem: análise da atuação do enfermeiro em centro cirúrgico [tese]. São Paulo (SP): Universidade de São Paulo; 1990.

2. Guido LA. Stress e coping entre enfermeiros de centro cirúrgico e recuperação anestésica [tese]. São Paulo (SP): Universidade de São Paulo; 2003.

3. Sangiuliano LA. Stress na atuação dos enfermeiros em um hospital privado e as consequências no seu estado de saúde [dissertação]. São Paulo (SP): Universidade de São Paulo; 2004.

4. Guarino AJ. Stress e capacitação de órgãos: uma realidade vivenciada pelos enfermeiros [dissertação]. São Paulo (SP): Universidade de São Paulo; 2005.

5. Batista, KM. Stress entre enfermeiros de unidade de emergência [dissertação]. São Paulo (SP): Universidade de São Paulo; 2005.

6. Menzani G. Stress entre enfermeiros brasileiros que atuam em pronto socorro [dissertação]. São Paulo (SP): Universidade de São Paulo; 2006.

7. Guerrer FJL. Estresse dos enfermeiros que atuam em unidades de terapia intensiva no Brasil [dissertação]. São Paulo (SP): Universidade de São Paulo; 2007.

8. Anabuki MH. Situações geradoras de estresse: a percepção das enfermeiras de um hospital de ensino [dissertação]. São Paulo (SP): Universidade de São Paulo; 2002.

9. Lima EDRP. Estresse ocupacional e a enfermagem de centro cirúrgico [dissertação]. Belo Horizonte (MG): Universidade Federal de Minas Gerais; 1997.

10. Krahl M. Prazer e o sofrimento no cotidiano do enfermeiro de centro cirúrgico [dissertação]. Florianópolis (SC): Universidade Federal de Santa Catarina; 2000.

11. Massaroni L. Estresse dos profissionais da equipe de enfermagem no centro cirúrgico: estudo de suas representações sociais [tese]. Rio de Janeiro (RJ): Universidade Federal do Rio de Janeiro; 2001.

12. Meirelles NF. Estresse ocupacional e o centro cirúrgico oncológico no contexto da enfermagem [dissertação]. Rio de Janeiro (RJ): Universidade Federal do Rio de Janeiro; 2002.

13. Aquino JM. Estressores no trabalho das enfermeiras em centro cirúrgico: conseqüências profissionais e pessoais [tese]. Ribeirão Preto (SP): Universidade de São Paulo; 2005.

14. Oliveira JS. Como as enfermeiras enfrentam o emergir do estresse no seu trabalho [dissertação]. Fortaleza 
(CE): Universidade Federal do Ceará; 1999.

15. Nunes MBG. Estresse nos trabalhadores de enfermagem: estudo em uma unidade de psiquiatria [dissertação]. Rio de Janeiro (RJ): Universidade do Estado do Rio de Janeiro; 2000.

16. Britto ES. Enfermeiros psiquiátricos: estresse, enfrentamento e saúde [dissertação]. Ribeirão Preto (SP): Universidade de São Paulo; 2006.

17. Silva JLL. Estresse e transtornos mentais comuns em trabalhadores de enfermagem [dissertação]. Rio de Janeiro (RJ): Universidade Federal do Estado do Rio de Janeiro; 2007.

18. Pafaro RC. Estudo do estresse do enfermeiro com dupla jornada de trabalho em um hospital de oncologia pediátrica de Campinas [dissertação]. Campinas (SP): Universidade Estadual de Campinas; 2002.

19. Ferreira LRC. Stress no cotidiano da equipe de enfermagem e sua correlação com o cronótipo [dissertação]. Campinas (SP): Universidade Estadual de Campinas; 2006.

20. Fernandes SMBA. Estresse ocupacional e o mundo do trabalho atual: repercussões na vida cotidiana das enfermeiras de uma instituição hospitalar pública -Natal/RN [dissertação]. Natal (RN): Universidade Federal do Rio Grande do Norte; 2005.

21. Boller E. Enfrentamento do estresse no trabalho da enfermagem em emergência: possibilidades e limites na implementação de estratégias gerenciais [dissertação]. Florianópolis (SC): Universidade Federal de Santa Catarina; 2002.

22. Gatti MFZ. Música como intervenção redutora da ansiedade do profissional de serviço de emergência: utopia ou realidade? [dissertação]. São Paulo (SP): Universidade de São Paulo; 2005.

23. Shigueno LYO. Exercício do banho de aspersão: um estudo sobre as práticas, as condições de trabalho e a saúde das trabalhadoras de enfermagem [dissertação]. São Paulo (SP): Universidade Federal de São Paulo; 2000.

24. Amaral TR. Dimensões psicossociais do trabalho da enfermagem e os distúrbios psíquicos menores em unidades críticas [dissertação]. Florianópolis (SC): Universidade Federal de Santa Catarina; 2006.

25. Ferreira FG. Desvendando o estresse da equipe de enfermagem em terapia intensiva [dissertação]. São
Paulo (SP): Escola de Enfermagem da Universidade de São Paulo; 1998.

26. Miranda AF. Estresse ocupacional: inimigo invisível do enfermeiro? [dissertação]. Ribeirão Preto (SP): Universidade de São Paulo; 1998.

27. Pegoraro IB. Níveis de stress em trabalhadores de unidades distritais de saúde: uma realidade preocupante [dissertação]. Ribeirão Preto (SP): Universidade de São Paulo; 2002.

28. Camelo SHH. Sintomas de estresse nos trabalhadores atuantes em cinco núcleos de saúde da família [dissertação]. Ribeirão Preto (SP): Universidade de São Paulo; 2002.

29. Camelo SHH. Riscos psicossociais relacionados ao estresse no trabalho das equipes de saúde da família e estratégias de gerenciamento [tese]. Ribeirão Preto (SP): Universidade de São Paulo; 2006.

30. Wai MFP. O trabalho do agente comunitário de saúde na Estratégia Saúde da Família: fatores de sobrecarga e mecanismos de enfrentamento [dissertação]. Ribeirão Preto (SP): Universidade de São Paulo; 2007. 